\title{
Critical Hypersurfaces and Instability FOR RECONSTRUCTION OF SCEnES in High Dimensional Projective Spaces
}

\author{
Marina Bertolini ${ }^{1}$, Luca Magri ${ }^{2}$ \\ ${ }^{1}$ Dipartimento di Matematica, \\ Università degli Studi di Milano, Milano, Italy \\ ${ }^{2}$ Dipartimento di Elettronica, Informazione e Bioingegneria (DEIB), \\ Politecnico di Milano, Milano, Italy \\ marina.bertolini@unimi.it
}

\begin{abstract}
In the context of multiple view geometry, images of static scenes are modeled as linear projections from a projective space $\mathbb{P}^{3}$ to a projective plane $\mathbb{P}^{2}$ and, similarly, videos or images of suitable dynamic or segmented scenes can be modeled as linear projections from $\mathbb{P}^{k}$ to $\mathbb{P}^{h}$, with $k>h \geq 2$. In those settings, the projective reconstruction of a scene consists in recovering the position of the projected objects and the projections themselves from their images, after identifying many enough correspondences between the images. A critical locus for the reconstruction problem is a configuration of points and of centers of projections, in the ambient space, where the reconstruction of a scene fails. Critical loci turn out to be suitable algebraic varieties. In this paper we investigate those critical loci which are hypersurfaces in high dimension complex projective spaces, and we determine their equations. Moreover, to give evidence of some practical implications of the existence of these critical loci, we perform a simulated experiment to test the instability phenomena for the reconstruction of a scene, near a critical hypersurface.
\end{abstract}

Key words: critical loci, projective reconstruction, computer vision, multiview geometry.

\section{Introduction}

As linear projections from $\mathbb{P}^{3}$ to $\mathbb{P}^{2}$ are the natural geometric model for images of static three-dimensional scenes captured with pinhole cameras, also linear projections from $\mathbb{P}^{k}$ to $\mathbb{P}^{h}$, with $k>h \geq 3$, can be useful in modelling images of particular dynamic and segmented scenes 10, 14, 17, 19,25, 26, 27. The classical problem of the reconstruction of a static scene - given multiple images of an unknown scene taken from unknown cameras, reconstruct the positions of cameras and of scene points - can be generalized as well in the setting of high dimensional projective spaces. These kinds of problems can be nicely reinterpreted with tools of projective algebraic geometry, which guarantee that sufficiently many images and sufficiently many sets of image correspondences allow for a successful projective reconstruction. The reader is referred to 15 for a wide overview of the role of projective geometry in Computer Vision. 
Nevertheless, even in the classical set up of two projections from $\mathbb{P}^{3}$ to $\mathbb{P}^{2}$ there are sets of critical points, i.e., points for which the projective reconstruction fails, in the sense that for each critical configuration of scene points there exist a non projectively equivalent sets of points and cameras that give the same images in the view planes.

The study of critical loci has been the object of interest for several authors, as shown in literature: in the case of a single view of a static scene, where the objective is only the reconstruction of the position of the camera and of the projection matrix (calibration), Buchanan, 8, showed that all the critical configurations lay on a twisted cubic curve. If the scene is static, it is well known that the minimum number of images necessary for a full reconstruction is two. For two views, quadric surfaces were shown to be critical hypersurfaces in 21,22]. In the case of three or more views, contributions are found in 16, 20, 23. A comprehensive, detailed analysis both in the case of two and in the case of multiple views was conducted in 13 .

The analysis of dynamic or segmented scenes has led to the study of projections from higher dimensional space $\mathbb{P}^{k}$ to the projective plane $\mathbb{P}^{2}$, as considered by Wolf and Shashua in 27, where the additional dimensions of $\mathbb{P}^{k}$, with respect to the ambient space, are used to encode information on the evolution of the scene. In this extended space the scene can be treated as static, providing a more manageable representation of dynamic or segmented scenes of the usual space. In this context, critical loci in the case of one view were theoretically described in [6]. The more involved analysis of the critical loci for projective reconstruction from multiple views in higher dimensions is approached in [7] and 2] where the general theoretical framework necessary to describe such critical loci is introduced. This framework showed that critical loci are special algebraic varieties, namely determinantal varieties, and in [3] the authors give a description of the critical loci as zero-sets of suitable ideals. More precisely, revisiting the previous framework in a fully projective context, in 3 critical loci for projective reconstruction in $\mathbb{P}^{k}$ from $n$ views to $\mathbb{P}^{2}$ turn out to be either hypersurfaces of degree $\frac{k+1}{2}=n$, if the ambient space is odd dimensional, or special determinantal varieties of codimension 2 and degree $\frac{(k+4)(k+2)}{8}$ if the ambient space is even dimensional, when $n$ is the minimum number of views necessary to allow the reconstruction.

Finally, the notion of criticality can naturally be extended to projections from $\mathbb{P}^{k}$ to image spaces of higher dimension, $\mathbb{P}^{h}, h \geq 3$, and the resulting critical loci turn out to be still determinantal varieties whose codimension in $\mathbb{P}^{k}$ depends on $k, h$ and on the number $n$ of projections.

In this paper the critical locus for $n$ projections from $\mathbb{P}^{k}$ to $\mathbb{P}^{h}, h \geq 3$ is studied under the hypothesis that $n$ is the minimal number of projections which allow the reconstruction of the scene, and the dimensions of the ambient space, $k$, and of the image space, $h$, are linked by the relation: $k \equiv h-1 \bmod h$. The interest for this case comes from the fact that, as shown in Section 3, under this numerical hypothesis the critical locus turns out to be a hypersurface in $\mathbb{P}^{k}$. 
This case can be also considered as a generalization of the situation studied in [3] when $k$ is odd. Indeed, in 3 projections are always performed on a projective plane $\mathbb{P}^{2}$, hence only static images can be modeled. While in this paper we consider projections on spaces of higher dimension $\mathbb{P}^{h}$ with $h \geq 3$. This generalization allows us to model videos of moving scenes, producing moving images in an image plane which can be treated as a static scene in a projective space of dimension $h \geq 3$.

Even if in our hypothesis the critical locus is a hypersurface hence it has the higher dimension allowed in the ambient space, from a practical point of view, it is almost unlikely that all points and all the cameras constitute a critical configuration. Nevertheless, for configuration close to critical ones, the attained reconstructions exhibit a certain degree of instability, in the sense that small perturbations of the image points change the reconstructed solution drastically. In order to validate this assertion, following the setup conceived in [7], a simulated experiment for projections $\mathbb{P}^{5} \rightarrow \mathbb{P}^{3}$ is performed.

The paper is organized as follows: in Section 2 notations are fixed, some basic definitions from projective algebraic geometry are recalled and a brief introduction to the general computer vision setting is offered for the convenience of the reader. In Section 3 the general theoretical framework for critical configurations and critical loci is described. Section 4 is dedicated to the study of the critical hypersurface: in particular its equation is determined and its singularities are investigated. In Section 5 the instability phenomena are shown in a particular case, i.e. for projections $\mathbb{P}^{5} \rightarrow \mathbb{P}^{3}$, with the help of Matlab [24].

\section{General results and preliminaries}

In this section we fix notation and terminology, we recall some definitions from projective algebraic geometry which will be useful in the sequel and we give a short overview of classical facts in computer vision related to the problem of projective reconstruction of scenes and cameras from multiple view.

\subsection{Notation and basic definitions from Algebraic Geometry}

Given a matrix $A=\left[a_{i j}\right]$ with real or complex entries, $A^{T}$ denotes its transpose. The $j$-th row of $A$ is denoted by $\mathbf{a}^{j}$. Moreover, $D R^{i_{1}, \ldots, i_{n}}(A)$ denotes the matrix obtained from $A$ by deleting rows $\mathbf{a}^{i_{1}}, \ldots, \mathbf{a}^{i_{n}}$.

If $\mathcal{A}$ is the set of the first $k$ integers $\{1,2,3, \ldots, k\}$, we denote by $\mathcal{A}^{\times n}$ the cartesian product of $\mathcal{A}$ with itself $n$-times, i.e. $\mathcal{A}^{\times n}=\mathcal{A} \times \cdots \times \mathcal{A}=\{1,2, \ldots, k\} \times \cdots \times\{1,2, \ldots, k\}$.

Now we give some basic definitions in Algebraic Geometry which are useful to understand the following sections. We shall limit ourselves to the case in which the ground field is the field of complex numbers, $\mathbb{C}$. However, for definitions and basic properties concerning projective algebraic varieties, we suggest, for example, [12] or [18. 
Following standard notation, $\mathbb{P}^{k}$ denotes the $k$-dimensional real (or complex) projective space and $\left(x_{1}, x_{1}, \ldots, x_{k+1}\right)$ the homogeneous coordinates of its points. Once a projective frame is chosen for $\mathbb{P}^{k}$, coordinate vectors $\mathbf{X}$ of points in $\mathbb{P}^{k}$ are written as columns, thus $\mathbf{X}^{T}=\left(X_{1}, X_{2}, \ldots, X_{k+1}\right)$. In this context, whenever multiplication by a non-zero scalar is utilized, the scalar will be real or complex, accordingly. A linear projective subspace $\Lambda \subseteq \mathbb{P}^{k}$ spanned by $m+1$ linearly independent points will be called $m$-space or subspace of dimension $m$. By convention the empty set is considered as a $(-1)$-space.

A projective algebraic variety in the projective space $\mathbb{P}^{n}$ is substantially a subset of points of $\mathbb{P}^{n}$ defined by the common zeros of a family of homogeneous polynomials.

We need some notions which are basic to study algebraic varieties. Their definitions need a certain amount of technical apparatus, hence we try to give here an informal approach, following 12 .

Given a homogenous ideal $I \subset \mathbf{C}\left[X_{1}, \ldots, X_{k+1}\right], V(I)$ denotes the projective algebraic variety defined as $V(I)=\left\{\mathbf{X} \in \mathbb{P}^{k}: f(\mathbf{X})=0\right.$ for all $\left.f \in I\right\}$. Details on this standard correspondence between ideals and varieties can be found for example in 9 .

An algebraic variety is said to be irreducible, if it cannot be expressed as the union of two non-empty proper sub-varieties. Every variety can be expressed as a finite union $\mathcal{X}=\mathcal{X}_{1} \cup \mathcal{X}_{2} \cup \ldots \cup \mathcal{X}_{r}$ of irreducible subsets (subvarieties) of $\mathcal{X}$ which are called irreducible components of $\mathcal{X}$.

The projective varieties contained in $\mathbb{P}^{n}$ are the closed sets of a topology called the Zariski topology of $\mathbb{P}^{n}$. The same name will be given to the topology induced by the Zariski topology on the subsets of $\mathbb{P}^{n}$.

In the Zariski topology, the non empty open sets are very big (they are dense), since the closed sets are the common zeros of some polynomials. This notion is necessary to introduce the notion of general (or, sometimes, generic). Indeed when a family $\left\{X_{p}\right\}_{p \in \Sigma}$ of objects (points, linear spaces, varieties,...) is parameterized by the points of an irreducible projective algebraic variety $\Sigma$, the expression "the general object of $\left\{X_{p}\right\}$ has the property $P$ " means that "the subset of points $p \in \Sigma$ such that the corresponding object $X_{p}$ has the property $P$, contains a Zariski open dense subset of $\Sigma$ " (see for example 12 , p. 53]). For example in the family of all the conics in $\mathbb{P}^{2}$, the general conic is irreducible, since the requirement to be degenerate corresponds to a closed condition in the Zariski topology of the family of conics.

The dimension, $\operatorname{dim}(\mathcal{X})$, of an irreducible projective variety $\mathcal{X}$ in $\mathbb{P}^{n}$ is the integer $k$ such that the general $n-k$-plane of $\mathbb{P}^{n}$ intersects $\mathcal{X}$ in a finite set of points.

If the variety is reducible, its dimension is the maximum of the dimensions of its irreducible components.

A projective variety defined by a single homogeneous polynomial is called an hypersurface of $\mathbb{P}^{n}$ and it has dimension $n-1$. A hypersurface $\mathcal{X}$ is the zero locus $V(I)$ if $I$ is a principal ideal, i.e., an ideal generated by only one element. 
The degree of a $k$-dimensional projective variety $\mathcal{X}$ in $\mathbb{P}^{n}$ is the number (with multiplicity) of points of intersection of $\mathcal{X}$ with a general $(n-k)$-plane of $\mathbb{P}^{n}$ (from the definition of dimension, this set of points is finite).

For instance a projective curve is a projective variety of dimension one. The degree of a projective curve in $\mathbb{P}^{3}$ is the number of intersection points of the curve with a generic 2-plane of $\mathbb{P}^{3}$.

A point $\mathbf{X}$ of an irreducible projective variety $\mathcal{X}$ defined by a family of homogenous polynomial $F_{i}$ is said to be singular if the Jacobian matrix $\left[\frac{\partial F_{i}}{\partial x_{j}}\right]$ has rank lower than maximum in $\mathbf{X}$. Otherwise the point is a smooth point. The variety $\mathcal{X}$ is smooth if it has no singular points. Notice that in a family of varieties the condition to be singular is a closed condition in the Zariski topology.

When the variety $\mathcal{X}$ is a hypersurface, a point $\mathbf{X} \in \mathcal{X}$ is singular if and only if each line passing through $\mathbf{X}$ intersects $\mathcal{X}$ with multiplicity bigger than 1 .

\subsection{General setting: scenes, cameras, views}

For the convenience of the reader, in this subsections we succinctly recall the concepts of pinhole cameras, centers of projection, views, reconstruction, and critical configurations. For more details we refer the reader to 15 for the classical case of scenes in $\mathbb{P}^{3}$, and to 2 for the general case of scenes in $\mathbb{P}^{k}$.

Given a scene, i.e., a set of points in the ambient $3 D$-space, the action of taking a picture can be modelled by maps that are linear projections from the space of the scene to the plane of the image, the so-called view. It is therefore very convenient and natural to assume that the ambient space is embedded in projective 3 -space $\mathbb{P}^{3}$ and, from the algebraic geometric point of view, it is more convenient to choose a complex ambient space, instead of the real one. Therefore, from now on, all projective spaces are assumed to be complex unless specifically mentioned.

A (pinhole) camera can be represented as a central projection $P$ of points in $\mathbb{P}^{3}$, from a point $C$, the center of the camera, onto the view plane $\mathbb{P}^{2}$. With respect to the homogeneous coordinates $\mathbf{X} \equiv\left(X_{1}, X_{2}, X_{3}, X_{4}\right)^{T}$ and $\mathbf{x} \equiv\left(x_{1}, x_{2}, x_{3}\right)^{T}$ in $\mathbb{P}^{3}$ and $\mathbb{P}^{2}$ respectively, the projection mapping $P: \mathbb{P}^{3} \backslash\{C\} \rightarrow \mathbb{P}^{2}$ can be described by $\mu \mathbf{x}=P \mathbf{X}$, where $\mu$ is a non-zero constant and the $3 \times 4$-matrix $P$ has maximal rank. The center of projection $C$ is the right annihilator of $P$. As customary, the projection map and one of its matrix representations in a chosen frame are identified. The set of points in $\mathbb{P}^{3}$ having the same image under projection $P$ is a line which is called a ray.

When several images of the same scene $\left\{\mathbf{X}_{j}\right\}$ are taken with different cameras $P_{i}$, $i=1, \ldots, n$, the images $\mathbf{x}_{i j}=P_{i}\left(\mathbf{X}_{j}\right), i=1, \ldots, n$ of the same point via different cameras are called corresponding points.

As mentioned above, several authors have introduced generalizations of the classical set up, dealing with certain types of dynamic or segmented scenes, that can be profitably 
modelled through the framework of multiple view geometry in higher dimensional spaces. In analogy with the situation in $\mathbb{P}^{3}$, a scene in $\mathbb{P}^{k}$ is a set of $N$ points $\left\{\mathbf{X}_{j}\right\} \in \mathbb{P}^{k}$. A camera is defined as a projection from $\mathbb{P}^{k}$ to a projective space $\mathbb{P}^{h}$ i.e. by a linear map $P$ associated to a full-rank $(k+1) \times(h+1)$ matrix $P$, whose null space is the center of projection. As before, a ray is the set of points that are mapped to the same point by $P$. In this case the center and the rays are linear subspaces of dimension $k-h-1$ and $k-h$ respectively. The notion of corresponding points generalizes to corresponding subspaces in the higher dimensional setting: proper linear subspaces $L_{i}, i=1 \ldots n$, of different views, are said to be corresponding if there exists at least a point $\mathbf{X} \in \mathbb{P}^{k}$ such that $P_{i}(\mathbf{X}) \in L_{i}$ for all $i=1 \ldots n$.

\subsection{Fundamental matrices and Grassmann tensors}

In the classical situation of two cameras $P_{1}$ and $P_{2}$ taking photographs of a scene $\left\{\mathbf{X}_{j}\right\} \subset \mathbb{P}^{3}$, the intrinsic relationships between corresponding points in the two view planes are summarized by a $3 \times 3$ matrix $F$ of rank 2 , the fundamental matrix associated to the pair of cameras $P_{1}$ and $P_{2}$. (see [15] for a thorough exposition.)

Generalizations of the notion of fundamental matrix for two view planes in $\mathbb{P}^{3}$, are given in two different ways. On one side, a generalized fundamental matrix is defined in [4] to express the relation between corresponding points in two image spaces $\mathbb{P}^{h_{i}}, i=1,2$, in $\mathbb{P}^{k}$. On the other side, Hartley and Schaffalitzky in [17], introduced a class of tensors, called Grassmann tensors, with the purpose of translating into appropriate equations the relationships among corresponding points, for multiple views in higher ambient spaces. As in the case of the fundamental matrix, Grassmann tensors are determined by the projection matrices and, vice versa, the projection matrices can be reconstructed from the Grassmann tensors, up to projective transformation of the ambient space. We recall here the basic elements of their construction and for more details see also 2, 17].

Consider a set of projections $P_{j}: \mathbb{P}^{k} \backslash C_{P_{j}} \rightarrow \mathbb{P}^{h_{j}}, j=1, \ldots, n, h_{j} \geq 2$ with centers in general position. Moreover consider a profile, i.e a partition $\left(\alpha_{1}, \alpha_{2}, \ldots, \alpha_{n}\right)$ of $k+1$, i.e. $1 \leq \alpha_{j} \leq h_{j}$ for all $j$, and $\sum \alpha_{j}=k+1$.

Let $\left\{L_{j}\right\}, j=1, \ldots, n$, where $L_{j} \subset \mathbb{P}^{h_{j}}$, be a set of general $s_{j}$-spaces, with $s_{j}=$ $h_{j}-\alpha_{j}$, and let $S_{j}$ be the maximal rank $\left(h_{j}+1\right) \times\left(s_{j}+1\right)$-matrix whose columns are a basis for $L_{j}$. By definition, if all the $L_{j}$ are corresponding subspaces there exists a point $\mathbf{X} \in \mathbb{P}^{k}$ such that $P_{j} \mathbf{X} \in L_{j}$ for $j=1, \ldots, n$. In other words, there exist $n$ vectors $\mathbf{v}_{j} \in \mathbb{C}^{s_{j}+1} j=1, \ldots, n$, such that:

$$
\left[\begin{array}{ccccc}
S_{1} & 0 & \ldots & 0 & P_{1} \\
0 & S_{2} & \ldots & 0 & P_{2} \\
\vdots & \vdots & \ddots & \vdots & \vdots \\
0 & \ldots & 0 & S_{n} & P_{n}
\end{array}\right]\left[\begin{array}{c}
\mathbf{v}_{1} \\
\mathbf{v}_{2} \\
\vdots \\
\mathbf{v}_{n} \\
\mathbf{X}
\end{array}\right]=\left[\begin{array}{c}
0 \\
0 \\
\vdots \\
0
\end{array}\right]
$$


The existence of a non trivial solution $\left\{\mathbf{v}_{1}, \ldots, \mathbf{v}_{n}, \mathbf{X}\right\}$ for system (1) implies that the system matrix has zero determinant. This determinant can be thought of as an $n$-linear form, i.e. a tensor, in the Plücker coordinates of the spaces $L_{j}$. This tensor is the Grassmann tensor. In the cases of two views the Grassmann tensor turns out to be the generalized fundamental matrix.

\subsection{Projective reconstruction}

While reconstruction problems can be posed in several geometric settings as metric, affine, or projective, this work is conducted entirely within the projective framework and therefore reconstruction will always be assumed to be achieved up to projective transformations.

Within a projective setting the camera center is the only property of the camera which is preserved under homographies of the view plane, hence projective reconstruction of cameras consists only of the determination of their centers.

In this subsection we are working under the assumption that the centers $C_{P_{j}}$ of the projections we consider are in general position. In the examples we will deal with, the technical assumption of centers being in general position implies that $\bigcap_{j} C_{P_{j}}=\emptyset$. Notice that reconstruction of a scene would be impossible if $\bigcap_{j} C_{P_{j}} \neq \emptyset$ because a scene-point $\mathbf{X}$ would be indistinguishable from any other point in the linear projective space generated by $\mathbf{X}$ and $\bigcap_{j} C_{P_{j}}$.

Given $n$ views of a scene $\left\{\mathbf{X}_{j}\right\} \subset \mathbb{P}^{k}$, the recovery of the scene structure has two consecutive stages: the reconstruction of the camera centers, followed by the reconstruction of the scene, i.e., the position of the points $\left\{\mathbf{X}_{j}\right\}$ in $\mathbb{P}^{k}$, once cameras have been determined.

To perform both these tasks one needs to have a sufficient number of corresponding points in a suitable number of views. In the classical case of $\mathbb{P}^{3}$, one easily sees that two views and eight corresponding points allow the reconstruction of the fundamental matrix $F$ by solving a linear system. Once $F$ is determined, projection matrices can also be reconstructed, 15, Section 8.5.3]

In the more general case of multiple views and higher dimensional spaces, reconstruction is significantly more involved and requires the use of Grassmann tensors, see 2,17 .

Assuming enough scene points are given, in general enough mutual positions, a first natural question is to determine the minimum number of views necessary to allow reconstruction. In this context two numbers play an important role: the minimum number $\omega_{k}$ of views necessary to reconstruct cameras $P_{i}$ and a minimum number $\mu_{k}$ of views necessary to reconstruct the scene $\left\{\mathbf{X}_{j}\right\}$ in $\mathbb{P}^{k}$, when the position of the centers are assumed to be known.

Both these numbers are implicitly given in [17], as the numerical conditions for the 
existence of a suitable Grassmann tensor, and explicitly computed in [2] under the hypothesis that all the image spaces have the same dimension $h$. For the convenience of the reader, we recall them.

Assume that the ambient space is $\mathbb{P}^{k}$ and all the target spaces have the same dimension, i.e. $h_{1}=h_{2}=\cdots=h_{n}=h$. Then the following propositions hold [2]:

Proposition 1. Assume $k-1=\sigma h+\lambda$, where $\sigma$ and $\lambda$ are non negative integers and $\lambda \leq h-1$. Assuming that cameras are known (up to projective equivalence), the minimum number of views necessary to reconstruct a scene for projections from $\mathbb{P}^{k}$ to $\mathbb{P}^{h}$ is

$$
\mu_{k, h}=\sigma+1
$$

Proposition 2. Assume $k=s h+l$, where $s$ and $l$ are non negative integers and $l \leq h-1$. The minimum number of views necessary to reconstruct the cameras for projections from $\mathbb{P}^{k}$ to $\mathbb{P}^{h}$ is

$$
\omega_{k, h}=s+1
$$

\subsection{Critical loci}

As discussed in the previous section, sufficiently many views and sufficiently many sets of corresponding points in the given views, should allow for a successful projective reconstruction. This is generally true, but it is very easy to notice that even in the classical set up of two projections from $\mathbb{P}^{3}$ to $\mathbb{P}^{2}$ one can have non projectively equivalent pairs of sets of scene points and cameras that produce the same images in the view planes, from a projective point of view, thus preventing reconstruction. Such configurations and the loci they describe are referred to as critical. Critical loci arising in the reconstruction from a single view, when only the camera can be reconstructed, are fully treated in [6]. A detailed treatment of critical loci in $\mathbb{P}^{3}$ is found in 13 , where the classical result of the criticality of a quadric surface in the case of 2-views, is analyzed.

A partial treatment of critical loci for multiple views in higher dimension is given in [2]. As mentioned in the introduction, in this paper a general framework to study critical loci was proposed, working in a setting in which affine charts had been chosen in each view. Critical loci were shown to be special determinantal varieties, and particular attention was given to the case of $\mathbb{P}^{4}$ in which a Bordiga surface was obtained as essential component of the critical locus. This case has been further investigated in a fully projective context in [5] and in 1]. Finally, critical loci for multiple views, i.e., for projections from $\mathbb{P}^{k}$ to $\mathbb{P}^{2}$, are extensively considered in 3 , where the varieties arising as critical loci turns out to be hypersurfaces of degree $r$ in $\mathbb{P}^{2 r-1}$ or varieties of codimension 2 and degree $\frac{(r+2)(r+1)}{2}$ in $\mathbb{P}^{2 r}$. Moreover, the ideal of these varieties is investigated.

We recall here the formalization of the notion of critical configuration and locus. Let us suppose to have $n$ views of a static scene in $\mathbb{P}^{k}$, consisting of a set of $N \geq k+3$ points $\left\{\mathbf{X}_{j}\right\}$ in $\mathbb{P}^{k}$. These $n$ views correspond to $n$ matrices $P_{i}, i=1, \ldots, n$, of dimension 
$(h+1) \times(k+1)$ and maximal rank which give the projections $\mathbf{x}_{i j}=P_{i}\left(\mathbf{X}_{j}\right)$ on the image $h$-spaces.

Definition 1. A set of points $\left\{\mathbf{X}_{j}\right\}, j=1, \ldots, N, N \geq k+3$, in $\mathbb{P}^{k}$ is said to be a critical configuration for projective reconstruction from $n$-views if there exist a non-projectively equivalent set of $N$ points $\left\{\mathbf{Y}_{j}\right\} \subset \mathbb{P}^{k}$ and two collections of $(h+1) \times(k+1)$ full-rank projection matrices $P_{i}$ and $Q_{i}, i=1, \ldots, n$, such that, for all $i$ and $j, P_{i} \mathbf{X}_{j}=\mu_{i j} Q_{i} \mathbf{Y}_{j}$, $\mu_{i j} \neq 0$. The two sets $\left\{\mathbf{X}_{j}\right\}$ and $\left\{\mathbf{Y}_{j}\right\}$ are called conjugate critical configurations, with associated conjugate matrices $\left\{P_{i}\right\}$ and $\left\{Q_{i}\right\}$.

According to [3], the natural setting to study the locus of all critical configurations associated to sets of conjugate matrices is the product variety $\mathbb{P}^{k} \times \mathbb{P}^{k}$, endowed with the two standard projections $\pi_{1}$ and $\pi_{2}$ onto the two factors.

Let $\left\{\mathbf{X}_{j}, \mathbf{Y}_{j}\right\}$ be conjugate critical configurations as above, with associated conjugate matrices $\left\{P_{i}\right\}$ and $\left\{Q_{i}\right\}$.

Definition 2. If $\left\{\left(\mathbf{X}_{j}, \mathbf{Y}_{j}\right)\right\}$ in $\mathbb{P}^{k} \times \mathbb{P}^{k}$ are pairs of conjugate critical configurations, with associated conjugate matrices $\left\{P_{i}\right\}$ and $\left\{Q_{i}\right\}$, the associated unified critical locus for projective reconstruction from n-views in $\mathbb{P}^{k} \times \mathbb{P}^{k}$ is the subscheme $\mathcal{U}^{k}=\mathcal{U}_{\left(\left\{P_{i}\right\},\left\{Q_{i}\right\}\right)}^{k} \subseteq$ $\mathbb{P}^{k} \times \mathbb{P}^{k}$ defined by the equations $P_{i} \mathbf{X}_{j}=\mu_{i j} Q_{i} \mathbf{Y}_{j}$, given in Definition 1 .

Critical loci appearing in practical applications, and studied in the literature, are the projections of $\mathcal{U}^{k}$ onto each factor. This motivates the following definition:

Definition 3. Let $\mathcal{U}^{k}$ be the unified critical locus for projective reconstruction from $n$-views with associated conjugate matrices $\left\{P_{i}\right\}$ and $\left\{Q_{i}\right\}$, and let $\pi_{1}$ and $\pi_{2}$ be the natural projection from $\mathbb{P}^{k} \times \mathbb{P}^{k}$ onto each factor. The corresponding critical locus and, respectively, conjugate critical locus for projective reconstruction from n-views in $\mathbb{P}^{k}$ are the subschemes:

$$
\mathcal{X}^{k}=\mathcal{X}_{\left(\left\{P_{i}\right\},\left\{Q_{i}\right\}\right)}^{k}=\pi_{1}\left(\mathcal{U}^{k}\right)
$$

or respectively

$$
\mathcal{Y}^{k}=\mathcal{Y}_{\left(\left\{P_{i}\right\},\left\{Q_{i}\right\}\right)}^{k}=\pi_{2}\left(\mathcal{U}^{k}\right)
$$

\section{The critical hypersurface in the case $k \equiv h-1 \bmod h$}

Explicit equations of the critical locus $\mathcal{X}^{k}$ can be obtained directly making use of the Grassmann tensor introduced in the previous section.

Indeed, the Grassmann tensor $\mathcal{T}^{P_{1}, \ldots, P_{n}}$ encodes the algebraic relations between corresponding subspaces in the different views of the projections $P_{1}, \ldots, P_{n}$. Hence by definition of critical set, if $\left\{\mathbf{X}_{j}, \mathbf{Y}_{j}\right\}$ are conjugate critical configurations, then, for each $j$, the projections $P_{1} \mathbf{X}_{j}, \ldots, P_{n} \mathbf{X}_{j}$ are corresponding points not only for the projections $P_{1}, \ldots, P_{n}$, but for the projections $Q_{1}, \ldots, Q_{n}$, too.

In this section we explicitly construct the Grassmann tensor for $n$ projections from $\mathbb{P}^{k}$ to $\mathbb{P}^{h}$, under the hypothesis that $k=h-1 \bmod h$. Then we use this tensor to 
get the generators of the ideal of $\mathcal{X}^{k}$, which, under our hypothesis, comes out to be a hypersurface.

The condition $k \equiv h-1 \bmod h$, together with the hypothesis that $n$ is the minimum number of views to get a reconstruction, implies that $k=n h-1$ and the only possible profile for the Grassmann tensor is $(h, h, \ldots, h)$.

Using the Grassmann formula we get that, if all the centers are in general position, $\operatorname{dim}\left(\bigcap_{j} C_{P j}\right)=k-n(h+1)<0$, hence the reconstruction is possible.

In this case $L_{1}, L_{2}, \ldots, L_{n}$ are points and equation (1) specializes to

$$
\underbrace{\left(\begin{array}{ccccc}
S_{1} & \mathbf{0} & \ldots & \mathbf{0} & P_{1} \\
\mathbf{0} & S_{2} & \ldots & \mathbf{0} & P_{2} \\
\vdots & \vdots & \ddots & \vdots & \vdots \\
\mathbf{0} & \mathbf{0} & \ldots & S_{n} & P_{n}
\end{array}\right)}_{T_{L_{1}, \ldots, L_{n}}^{P_{1}, \ldots, P_{n}}}\left(\begin{array}{c}
\lambda_{1} \\
\lambda_{2} \\
\vdots \\
\lambda_{n} \\
\mathbf{X}
\end{array}\right)=\mathbf{0}
$$

where $S_{j}=\left(x_{1, j}, \ldots, x_{h+1, j}\right)^{T}$ are the homogeneous coordinates of points $L_{j}$. The left matrix $T_{L_{1}, \ldots, L_{n}}^{P_{1}, \ldots, P_{n}}$ becomes a square one of dimension $n(h+1) \times n(h+1)$. If in addition $L_{1}, \ldots, L_{n}$ are corresponding points, the above linear system has a nontrivial solution $\left\{\lambda_{1}, \ldots, \lambda_{n}, \mathbf{X}\right\}$ and therefore

$$
\operatorname{det}\left(T_{L_{1}, \ldots, L_{n}}^{P_{1}, \ldots, P_{n}}\right)=0 .
$$

Moreover, the case $\mathbf{X}=\mathbf{0}$ doesn't occur. Otherwise, there would exist a certain $\bar{i}$ for which $\lambda_{\bar{i}} \neq 0$ and we could get $\mathbf{0}=P_{i} \mathbf{X}=\lambda_{\bar{i}} \mathbf{x}_{\bar{i}}$ which implies $\mathbf{x}_{\bar{i}}=\mathbf{0}$, a contradiction.

In other words, for the chosen profile $(h, \ldots, h)$, one sees that $\operatorname{det}\left(T_{L_{1}, \ldots, L_{n}}^{P_{1}, \ldots, P_{n}}\right)=0$ is indeed the $n$-linear constraint between the homogeneous coordinates $\left(x_{1, j}, \ldots, x_{h+1, j}\right)$ of the points $L_{j}$ so to let them be correspondent.

Analogously, if $L_{1}^{\prime}, \ldots, L_{n}^{\prime}$ is a set of corresponding points in $n$ views, for a set of projections $Q_{1}, \ldots, Q_{n}$, we get:

$$
\underbrace{\left(\begin{array}{ccccc}
S_{1}^{\prime} & \mathbf{0} & \ldots & \mathbf{0} & Q_{1} \\
\mathbf{0} & S_{2}^{\prime} & \ldots & \mathbf{0} & Q_{2} \\
\vdots & \vdots & \ddots & \vdots & \vdots \\
\mathbf{0} & \mathbf{0} & \ldots & S_{n}^{\prime} & Q_{n}
\end{array}\right)}_{T_{L_{1}^{\prime}, \ldots, L_{n}^{\prime}}^{Q_{1}, \ldots, Q_{n}}}\left(\begin{array}{c}
\lambda_{1} \\
\lambda_{2} \\
\vdots \\
\lambda_{n} \\
\mathbf{X}
\end{array}\right)=\mathbf{0}
$$

and the $n$ linear relation between $L_{1}^{\prime}, \ldots, L_{n}^{\prime}$ is given by the vanishing of $\operatorname{det}\left(T_{L_{1}^{\prime}, \ldots, L_{n}^{\prime}}^{Q_{1}, \ldots, Q_{n}}\right)$.

Considering as corresponding spaces $L_{1}^{\prime}=P_{1} \mathbf{X}, \ldots, L_{n}^{\prime}=P_{n} \mathbf{X}$, expressed in coordinates as $S_{j}^{\prime}=\left(\mathbf{p}_{j}^{1} \mathbf{X}, \ldots, \mathbf{p}_{j}^{h+1} \mathbf{X}\right)^{T}$, with $\mathbf{X}$ any point in the critical locus, one gets that 
the determinant of the following matrix must vanish:

$$
M^{\prime}=\left(\begin{array}{ccccc}
\mathbf{p}_{1}^{1} \mathbf{X} & & & & \\
\vdots & \mathbf{0} & \ldots & \mathbf{0} & Q_{1} \\
\mathbf{p}_{1}^{h+1} \mathbf{X} & & & & \\
& \mathbf{p}_{2}^{1} \mathbf{X} & & & \\
\mathbf{0} & \vdots & \ldots & \mathbf{0} & Q_{2} \\
& \mathbf{p}_{2}^{h+1} \mathbf{X} & & & \\
\vdots & \vdots & \ddots & \vdots & \vdots \\
& & & \mathbf{p}_{n}^{1} \mathbf{X} & \\
\mathbf{0} & \mathbf{0} & \ldots & \vdots & Q_{n} \\
& & & \mathbf{p}_{n}^{h+1} \mathbf{X} &
\end{array}\right) .
$$

Hence the determinant of $M^{\prime}$ generates the ideal of the critical locus $\mathcal{X}^{k}$, as $\mathbf{X}$ has to satisfy no other constraint. So we get that the ideal is principal and we have got the following:

Theorem 1. Let $k=n h-1$ and let $n$ be the number of views. Then $\mathcal{X}^{k}$ is a hypersurface of degree $n$, whose equations is

$$
g=\sum_{\left(j_{1}, \ldots j_{n}\right) \in \mathcal{A}^{\times n}} \mathbf{p}_{1}^{j_{1}} \mathbf{X} \cdots \mathbf{p}_{n}^{j_{n}} \mathbf{X} \operatorname{det}\left(D R^{j_{1}, \ldots, j_{n}}(\mathcal{Q})\right),
$$

where $\mathcal{Q}$ is the $n(h+1) \times(k+1)$ matrix given by staking in column the projection matrices $Q_{j}$ :

$$
\mathcal{Q}=\left(\begin{array}{c}
Q_{1} \\
Q_{2} \\
\vdots \\
Q_{n}
\end{array}\right)
$$

As already noted in the Introduction, it is worth observing that the case analysed in Theorem 1 is a generalization to projections to $\mathbb{P}^{h}, h \geq 3$, of the case, discussed in $\left[3\right.$. Section 4] of projections on $\mathbb{P}^{2}$. Indeed the equation 6 obtained above for the hypersurface $\mathcal{X}^{k}$ is analogous to the one computed in [3, equation (8)], but the techniques used are very different. Indeed the procedure followed in 3 is much more involved as it conducts to get the generators of the principal ideal via the study of the actions of goups on the maximal minors of suitable matrices. While here we get the generator of the principal ideal via a direct application of the Grassmann tensor. 


\section{Singularities of the hypersurface $\mathcal{X}^{k}$}

In this section we investigate the singularities of $\mathcal{X}^{k}$ and we prove the following proposition:

Proposition 3. The points of $\mathbb{P}^{k}$ which belongs to at least two center of projections are singular points for the hypersurface $\mathcal{X}^{k}$, in other words

$$
\bigcup_{i, j=1 \ldots n}\left(C_{P_{i}} \cap C_{P_{j}}\right) \subset \mathcal{F}^{k}
$$

where $C_{P_{i}}$ and $C_{P_{j}}$ denotes the centers of the projections $P_{i}$ and $P_{j}$, respectively, and $\mathcal{F}^{k}$ denotes the singular locus of $\mathcal{X}^{k}$. Moreover if $k \geq 2(h+1)$ then $\mathcal{F}^{k} \neq \emptyset$, hence $\mathcal{X}^{k}$ is singular.

Proof. The thesis holds for a generic hypersurface of equation

$$
f=\sum_{\left(j_{1}, \ldots j_{n}\right) \in \mathcal{A} \times n} a_{j_{1}, \ldots j_{n}} \mathbf{p}_{1}^{j_{1}} \mathbf{X} \ldots \mathbf{p}_{n}^{j_{n}} \mathbf{X}
$$

where the coefficients $a_{j_{1}, \ldots j_{n}} \in \mathbb{C}$ are not all zero. Indeed, the structure of the coefficients $a_{j_{1}, \ldots j_{n}}$, which for the equation of $\mathcal{X}^{k}$ are the maximal minors of the matrix $\mathcal{Q}$, is not relevant for the implication of the proposition; hence in the following we will consider a hypersurface $V(f)$ for arbitrary coefficients $a_{j_{1}, \ldots j_{n}}$.

First we can notice that all the projection centers $C_{P_{i}}, i=1 \ldots n$, lies on $V(f)$. Indeed each $C_{P_{i}}$ is a $(k-h-1)$-linear subspace of $\mathbb{P}^{k}$, given by

$$
C_{P_{i}}=\bigcap_{j=1 \ldots h+1} V\left(\mathbf{p}_{i}^{j} \mathbf{X}\right)
$$

and, for each fixed $i$, every summand of $f$ contains one $\mathbf{p}_{i}^{j} \mathbf{X}$ as a factor.

Then we show that $C_{P_{i}} \cap C_{P_{j}} \subseteq \mathcal{F}^{k}$, for each $i, j=1 \ldots n, i \neq j$. Indeed, $\mathcal{F}^{k}$ is the set of points $\bar{Y} \in \mathcal{X}^{k}$ such that each line passing through $\bar{Y}$ intersects $\mathcal{X}^{k}$ with multiplicity bigger than 1 .

Let $l=<\bar{Y}, \bar{Z}>=\left\{\lambda \bar{Y}+\mu \bar{Z} \mid(\lambda: \mu) \in \mathbb{P}^{1}\right\}$ a line through $\bar{Y}$; the intersection points $l \cap \mathcal{X}^{k}$ are computed via the solutions $\lambda$ and $\mu$ of the equation: 


$$
\begin{array}{r}
\sum_{\left(j_{1}, \ldots j_{n}\right) \in \mathcal{A}^{\times n}} a_{j_{1}, \ldots j_{n}} \mathbf{p}_{1}^{j_{1}}(\lambda \bar{Y}+\mu \bar{Z}) \ldots \mathbf{p}_{n}^{j_{n}}(\lambda \bar{Y}+\mu \bar{Z})= \\
\sum_{\left(j_{1}, \ldots j_{n}\right) \in \mathcal{A}^{\times n}} a_{j_{1}, \ldots j_{n}}\left(\lambda \mathbf{p}_{1}^{j_{1}}(\bar{Y})+\mu \mathbf{p}_{1}^{j_{1}}(\bar{Z})\right) \ldots\left(\lambda \mathbf{p}_{n}^{j_{n}}(\bar{Y})+\mu \mathbf{p}_{n}^{j_{n}}(\bar{Z})\right)= \\
\lambda^{n} \sum_{\left(j_{1}, \ldots j_{n}\right) \in \mathcal{A}^{\times n}} a_{j_{1}, \ldots j_{n}} \mathbf{p}_{1}^{j_{1}}(\bar{Y}) \ldots \mathbf{p}_{n}^{j_{n}}(\bar{Y}) \\
+\lambda^{n-1} \mu \sum_{\left(j_{1}, \ldots j_{n}\right) \in \mathcal{A}^{\times n}} a_{j_{1}, \ldots j_{n}} \mathbf{p}_{1}^{j_{1}}(\bar{Y}) \ldots \mathbf{p}_{s}^{j_{s}}(\bar{Z}) \ldots \mathbf{p}_{n}^{j_{n}}(\bar{Y}) \\
\sum_{a_{j_{1}, \ldots j_{n}} \mathbf{p}_{1}^{j_{1}}(\bar{Y}) \ldots \mathbf{p}_{s}^{j_{s}}(\bar{Z}) \ldots \mathbf{p}_{t}^{j_{t}}(\bar{Z}) \ldots \mathbf{p}_{n}^{j_{n}}(\bar{Y})}+\ldots=0,
\end{array}
$$

with $(\lambda, \mu) \neq(0,0)$ and $s, t=1, \ldots, n$.

Obviously we get that $\bar{Y} \in \mathcal{F}^{k} \Longleftrightarrow \mu=0$ is a double solution of $(9) \Longleftrightarrow$ the coefficient of $\lambda^{n-1} \mu$ vanishes for all $\bar{Z}$, being the coefficient of $\lambda^{n}$ zero, as $\bar{Y} \in \mathcal{X}^{k}$. If $\bar{Y}$ belongs to at least two centers, this condition is verified.

Moreover, computing the dimension of $C_{P_{i}} \cap C_{P_{j}}$ in $\mathbb{P}^{k}$ via the Grassmann formula, we get that $\operatorname{dim}\left(C_{P_{i}} \cap C_{P_{j}}\right) \geq 0$ if and only if $k \geq 2(h+1)$, hence, under this assumption, the hypersurface $k \geq 2(h+1)$ is singular.

\section{Experimental validation and instability results}

This section is devoted at reporting numerical results to demonstrate the occurrence of instability phenomena near critical loci. Although, from a practical point of view, it is almost unlikely that all points and all the cameras constitute a critical configuration, nevertheless, for configuration close to critical ones, the attained reconstructions exhibit a certain degree of instability, in the sense that small perturbations of the points change the reconstructed solution drastically.

In order to validate the above discussion, following the setup conceived in [7], an experiment for projections $\mathbb{P}^{5} \rightarrow \mathbb{P}^{3}$ is performed. Specifically, we illustrate the instability of the reconstruction of a dynamic scene modelled by two projections from $\mathbb{P}^{5}$ to $\mathbb{P}^{3}$ by performing the following steps.

- Random generation of projection matrices

Two pairs of projections matrices $\left\{P_{1}, P_{2}\right\},\left\{Q_{1}, Q_{2}\right\}$ are instantiated: without loss of generality $P_{1}$ is chosen as the canonical projection, the remaining projections are randomly generated with integer entries: 


$$
\begin{aligned}
& P_{1}=\left(\begin{array}{cccccc}
1 & 0 & 0 & 0 & 0 & 0 \\
0 & 1 & 0 & 0 & 0 & 0 \\
0 & 0 & 1 & 0 & 0 & 0 \\
0 & 0 & 0 & 1 & 0 & 0
\end{array}\right), \quad P_{2}=\left(\begin{array}{cccccc}
-3 & 0 & 0 & -1 & 2 & 1 \\
-1 & -1 & 0 & 1 & 0 & 0 \\
0 & 3 & -2 & 0 & 0 & -1 \\
3 & 2 & -2 & 2 & 0 & 0
\end{array}\right), \\
& Q_{1}=\left(\begin{array}{cccccc}
0 & -1 & 1 & 1 & 0 & 0 \\
1 & 0 & 0 & 0 & 1 & -2 \\
0 & 3 & 0 & 0 & -1 & 0 \\
0 & 2 & 1 & -2 & -1 & -1
\end{array}\right), \quad Q_{2}=\left(\begin{array}{cccccc}
0 & 2 & 0 & 0 & 1 & -4 \\
-1 & 1 & 2 & 1 & 0 & 0 \\
1 & 0 & 0 & 1 & 0 & 0 \\
2 & 1 & -1 & 0 & -2 & -1
\end{array}\right) \text {. }
\end{aligned}
$$

\section{- Equations of the critical locus}

The ideal of the critical locus for the projection matrices $\left\{P_{1}, P_{2}\right\},\left\{Q_{1}, Q_{2}\right\}$ is determined, using MACAULAY2 [11].

\section{- Random generation of critical points}

A set $X$ of 500 points on the critical loci is randomly generated; this set of critical points on the corresponding algebraic set was obtained regarding the defining polynomial as a real valued function and finding randomly distributed zeros through numerical routines in MATLAB $24[1]$.

\section{- Perturbation of the critical points}

The points in $X$ are perturbed with increasing levels of zero-mean gaussian noise, in particular we considered several levels of standard deviation $\sigma_{j}$, and obtained various perturbed configurations. Precisely, we considered 30 different values of standard deviation logarithmically spaced between decades $10^{-16}$ and $10^{-14}$.

\section{- Projection of the perturbed critical points}

For each perturbed configuration, i.e. for each $\sigma_{j}, j=1, \ldots, 30$, the noisy configurations of points are projected in $\mathbb{P}^{3}$ using the camera matrices previously introduced.

\section{- Fundamental matrix estimation}

Critical points are projected on the two views giving rise to pairs of corresponding points. These correspondence are hence used to estimate a generalized fundamental matrix $F_{\text {points }}$. The procedure [4] employed to compute $F_{\text {points }}$ follow closely the classical one to estimate the fundamental matrix in the case of projections from $\mathbb{P}^{3}$ and $\mathbb{P}^{2}$ : every pair of corresponding points give rise to a constraint on the entries of $F_{\text {points }}$ which in turn are determined by solving an overdetermined linear system. As a reference, a generalized fundamental matrix $F_{\text {cams }}$ was also computed directly from the cameras. This matrix is not affected by the instability phenomenon.

\footnotetext{
${ }^{1}$ Code available upon request.
} 


\section{- Estimating instability}

Finally we compare the fundamental matrix obtained from correspondences with the one computed from cameras. In order to assess the quality of the reconstructions computed from the perturbed point clouds, we compared the two fundamental matrices, measuring their antipodal distance $d\left(F_{\text {points }}, F_{\text {cams }}\right)$. In other words, as both $F_{\text {points }}$ and $F_{\text {cams }}$ are defined up to a multiplicative factor, we identified the space of generalized fundamental matrices with a quotient of the unit sphere in $\mathbb{R}^{16}$ and evaluate the distance between the corresponding two points as:

$$
d(A, B)=\min \{\|A-B\|,\|A+B\|\}
$$

\section{- Displaying the results}

The distribution of these distances with respect to the noise level is reported in Figure 1, where the average angular distance in 1000 trials is reported for each $\sigma_{j}$.

It can be appreciated that when the points of the scene lie near the critical locus - i.e. low values of noise - the instability of the reconstruction ends in the fact that $F_{\text {points }}$ is far from $F_{\text {cams }}$ and their respective distance are affected by great variance. Therefore, the flawed estimation of $F_{\text {points }}$ determines an unreliable reconstruction of a point cloud close to be critical. On the contrary, when the points are far away from the neighborhood

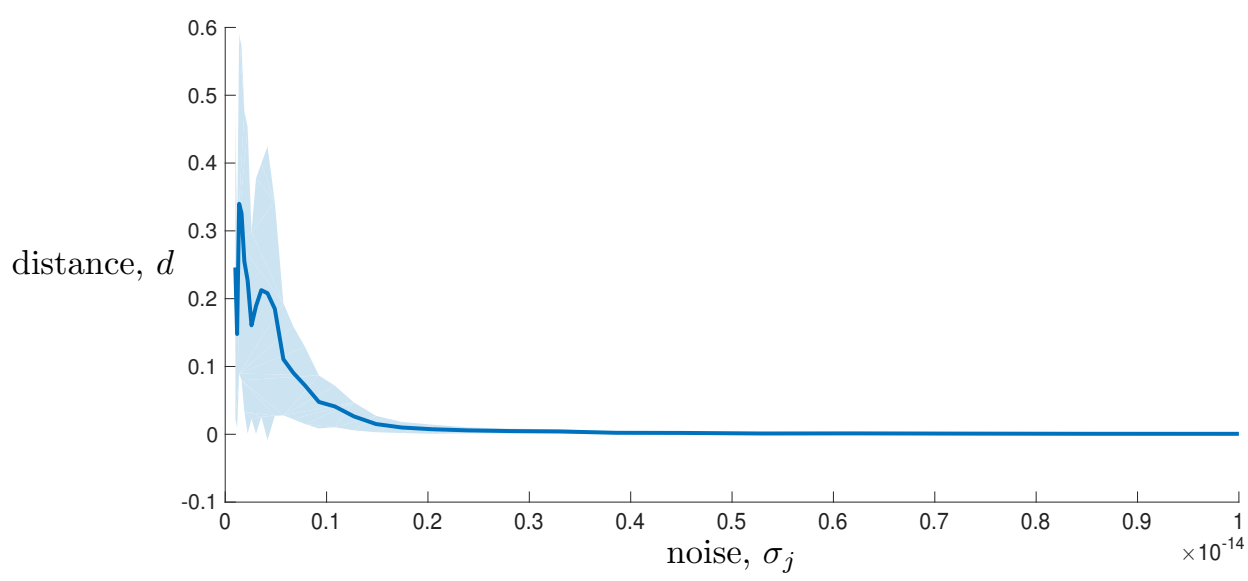

Fig. 1. The antipodal distance $d\left(F_{\text {points }}, F_{\text {cams }}\right)$, with respect to different levels of noise $\sigma_{j}$, in points on a critical configuration. The average distance is the blue line plot, the width of the shadowed area corresponds to \pm standard deviation of the antipodal distances distribution. 
of the critical locus - high values of $\sigma$ - the fundamental matrix $F_{\text {points }}$ estimated from the correspondences is consistently close to the reference $F_{\text {cams }}$, and can be profitably used to start the reconstruction process.

This phenomenon is absolutely consistent with the situations analyzed in the other papers 2, 6, 7]: as expected, the larger the distance of points from the critical locus is, the stabler the reconstruction gets.

\section{Conclusion}

In this paper we study the critical locus for the projective reconstruction of a set of points, in the case of $n$ projections from $\mathbb{P}^{k}$ to $\mathbb{P}^{h}$ for $k>h \geq 3$, where $n$ is the minimum number of projections which allows the reconstruction (Propositions 1 and 2 and the dimensions of the ambient space, $k$, and of the image space, $h$, are linked by the relation: $k \equiv h-1 \bmod h$. Under this numerical hypothesis (Section 3 ) the critical locus turns out to be a hypersurface in the ambient space, hence it has the higher dimension allowed. The main theoretical result of the paper is contained in Section 3, where, using the notion of Grassmann tensors previously recalled, the equation of the critical hypersurface is obtained in Theorem 1 .

Finally, to give evidence of some practical implications of the existence of critical loci, we perform a simulated experiment, in the case of two projections from $\mathbb{P}^{5}$ to $\mathbb{P}^{3}$, to show the instability phenomena for the reconstruction of a scene near a critical hypersurface. Indeed, for points close to the critical locus, the attained reconstruction exhibits a certain degree of instability, in the sense that small perturbations of the points change the reconstructed solution drastically.

\section{Acknowledgement}

The authors would like to thank Cristina Turrini for helpful conversations.

\section{References}

[1] M. Bertolini, G. Besana, R. Notari, and C. Turrini. Critical loci in computer vision and matrices dropping rank in codimension one. J. of Pure and Applied Algebra, 224(12):106439, 2020. doi $10.1016 /$ j.jpaa.2020.106439

[2] M. Bertolini, G. Besana, and C. Turrini. Applications of multiview tensors in higher dimensions. In S. Aja-Fernández, R. de Luis García, D. Tao, and X. Li, editors, Tensors in image processing and computer vision, Advances in Pattern Recognition, pages 237-260. Springer, London, 2009. doi 10.1007/978-1-84882-299-3_11

[3] M. Bertolini, G. Besana, and C. Turrini. Critical loci for projective reconstruction from multiple views in higher dimension: A comprehensive theoretical approach. Linear Algebra and its Applications, 469:335-363, 2015. doi 10.1016/j.laa.2014.11.021 
[4] M. Bertolini, G. Besana, and C. Turrini. Generalized fundamental matrices as grassmann tensors. Annali di Matematica Pura ed Applicata (1923 -), Jul 2016. doi 10.1007/s10231-016-0585-4

[5] M. Bertolini, R. Notari, and C. Turrini. The bordiga surface as critical locus for 3-view reconstructions. J. of Symbolic Computation, 91:74 - 97, 2019. MEGA 2017, Effective Methods in Algebraic Geometry, Nice (France), June 12-16, 2017. doi 10.1016/j.jsc.2018.06.014

[6] M. Bertolini and C. Turrini. Critical configurations for 1-view in projections from $\mathbb{P}^{k} \rightarrow \mathbb{P}^{2}$. J. of Mathematical Imaging and Vision, 27:277-287, 2007. doi 10.1007/s10851-007-0649-6

[7] M. Bertolini, C. Turrini, and G. Besana. Instability of projective reconstruction of dynamic scenes near critical configurations. In IEEE Int. Conf. on Computer Vision, pages 1-7, Los Alamitos, CA, USA, 2007. IEEE Computer Society. doi 10.1109/ICCV.2007.4409100

[8] T. Buchanan. The twisted cubic and camera calibration. Computer Vision, Graphics, and Image Processing, 42(1):130-132, 1988. doi 10.1016/0734-189X(88)90146-6

[9] D. A. Cox, J. Little, and D. O'Shea. Ideals, Varieties, and Algorithms: An Introduction to Computational Algebraic Geometry and Commutative Algebra. Springer, 2015. doi 10.1007/978-3-31916721-3

[10] X. Fan and R. Vidal. The space of multibody fundamental matrices: Rank, geometry and projection. In R. Vidal, A. Heyden, and Y. Ma, editors, Dynamical Vision. Proc. Int. Workshop on Dynamical Vision WDV 2005, volume 4358 of Lecture Notes in Computer Science, pages 1-17. Springer, 2007. doi $10.1007 / 978-3-540-70932-9 \_1$

[11] D. R. Grayson and M. Stillman. Macaulay2, a software system for research in algebraic geometry. http://macaulay2.com

[12] J. Harris. Agebraic Geometry: A First Course, volume 133 of Graduate Texts in Mathematics. Springer-Verlag, New York, 1992. doi 10.1007/978-1-4757-2189-8.

[13] R. Hartley and F. Kahl. Critical configurations for projective reconstruction from multiple views. Int. J. of Computer Vision, 71(1):5-47, 2007. doi 10.1007/s11263-005-4796-1

[14] R. Hartley and R. Vidal. The multibody trifocal tensor: motion segmentation from 3 perspective views. In Proc. 2004 IEEE Computer Society Conf. on Computer Vision and Pattern Recognition CVPR 2004, volume 1, pages I-I, Washington, DC, USA, 27 Jun-2 Jul 2004. doi $10.1109 /$ CVPR.2004.1315109

[15] R. Hartley and A. Zisserman. Multiple View Geometry in Computer Vision. Cambridge University Press, New York, 2nd edition, 2003. doi 10.1017/CBO9780511811685

[16] R. I. Hartley. Ambiguous configurations for 3-view projective reconstruction. In Computer Vision. Proc. European Conf. on Computer Vision ECCV 2000, Part I, volume 1842 of Lecture Notes in Computer Science, pages 922-935, Dublin, Ireland, 26 Jun-1 Jul 2000. Springer. doi 10.1007/3-54045054-8_60

[17] R. I. Hartley and F. Schaffalitzky. Reconstruction from projections using Grassmann tensors. Int. J. of Computer Vision, 83(3):274-293, 2009. doi 10.1007/s11263-009-0225-1.

[18] R. Hartshorne. Algebraic Geometry, volume 52 of Graduate Texts in Mathematics. Springer, New York, 1977. doi 10.1007/978-1-4757-3849-0

[19] K. Huang, R. Fossum, and Y. Ma. Generalized rank conditions in multiple view geometry with applications to dynamical scenes. In Computer Vision. Proc. European Conf. on Computer Vision ECCV 2002, Part II, volume 2351 of Lecture Notes in Computer Science, pages 201-216, Copenhagen, Denmark, 28-31 May 2002. Springer. doi 10.1007/3-540-47967-8_14

[20] F. Kahl, R. Hartley, and K. Astrom. Critical configurations for n-view projective reconstruction. In 
Proc. IEEE Computer Society Conf. on Computer Vision and Pattern Recognition CVPR 2001, pages II-II, Kauai, HI, USA, 8-14 Dec 2001. doi 10.1109/CVPR.2001.990945

[21] J. Krames. Zur Ermittlung eines Objektes aus zwei Perspektiven (Ein Beitrag zur Theorie der ,,gefährlichen Örter”). Monatshefte für Mathematik und Physik, 49:327-354, 1940. doi $10.1007 / \mathrm{BF} 01707311$

[22] S. Maybank. Theory of Reconstruction from Image Motion, volume 28 of Springer Series in Information Sciences. Springer, Berlin, Heidelberg, 1993. doi 10.1007/978-3-642-77557-4.

[23] A. Shashua and S. J. Maybank. Degenerate $n$ point configurations of three views: Do critical surfaces exist? Technical Report TR 96-19, Hebrew University, 1996. http://www.cs.huji.ac.il/ $\sim$ shashua/papers/cvpr-critical.ps.gz

[24] The MathWorks, Inc. MAtLAB. Natick, MA, USA. https://www.mathworks.com

[25] R. Vidal and Y. Ma. A unified algebraic approach to 2-D and 3-D motion segmentation and estimation. J. of Mathematical Imaging and Vision, 25(3):403-421, 2006. doi 10.1007/s10851-006$8286-\mathrm{z}$

[26] R. Vidal, Y. Ma, S. Soatto, and S. Sastry. Two-view multibody structure from motion. Int. J. of Computer Vision, 68(1):7-25, 2006. doi 10.1007/s11263-005-4839-7

[27] L. Wolf and A. Shashua. On projection matrices $\mathcal{P}^{k} \rightarrow \mathcal{P}^{2}, k=3, \ldots, 6$, and their applications in computer vision. Int. J. of Computer Vision, 48(1):53-67, 2002. doi 10.1023/A:1014855311993

Marina Bertolini is currently Associate Professor of Geometry at the Department of Mathematics at the Università degli Studi di Milano, Italy. Her main field of research is Complex Projective Algebraic Geometry, with particular interest for the classification of projective varieties and for the geometry of Grassmmann varieties. In the last years she has started to work also on applications of Algebraic Geometry to Computer Vision problems, and, in particular, to critical loci for reconstruction and to the geometric properties of multi-view tensors.

Luca Magri graduated in Mathematics at the University of Milan (IT) in 2012. In 2015, he received the $\mathrm{PhD}$ from the University of Milan with a thesis on robust multiple model fitting for Computer Vision applications. From 2015 to 2018, he has been a post-doc researcher first at the University of Verona (Dept. of Computer Science) and then at the University of Udine (DPIA), in these periods he collaborated with 3DFlow srl on 3D reconstruction themes. In 2018-2019 he joined the R\&D group of FARO Technologies (Rezzato, IT) where he worked on acquisition and registration techniques for structured light scanners. Currently it is at Dipartimento di Elettronica, Informazione e Bioingegneria of the Politecnico di Milano (DEIB) as a postdoctoral researcher. 